\title{
Direct lineage conversion: induced neuronal cells and induced neural stem cells
}

\author{
Zixiao Shi, Jianwei Jiao \\ Institute of Zoology, State Key Laboratory of Reproductive Biology, Chinese Academy of Sciences, Beijing 100101, China \\ $\triangle$ Correspondence: jwjiao@ioz.ac.cn \\ Received July 26, 2012 Accepted August 23, 2012
}

\begin{abstract}
Cellular reprogramming to neural cells is an area of ongoing study in developmental neuroscience, and recent research has generated remarkable achievements. Several studies have shown that the ectopic expression of specific neural transcription factors can convert terminally differentiated cells into neural cells. Here, we review the most recent progress in the field of induced neuronal (iN) cells and induced neural stem (iNS) cells and their potential clinical applications.
\end{abstract}

KEYWORDS iN cells, iNS cells, conversion, reprogramming, neuron, fibroblast

\section{INTRODUCTION}

The initial example of reprogramming mediated by overexpression of transcription factors can be traced back to experiments in which $\mathrm{MyoD}$, a master muscle cell regulatory transcription factor, was shown to activate many muscle-specific genes of in fibroblasts in the 1980s. However, it was presumed that this activation was possible only in related restricted cell lineages (Davis et al., 1987). For decades, it was considered that cellular differentiation and lineage commitment during development were robust and irreversible, but the generation of induced pluripotent stem (iPS) cells completely upended this model. In 2006, Takahashi and Yamanaka showed that mouse fibroblasts can be reprogrammed into iPS cells with a combination of four transcription factors (Yamanaka and Takahashi, 2006). This raised the question of whether defined transcription factors could also directly induce somatic cells into cell types of cells other than iPS cells. In 2010, several groups reported successful lineage reprogramming from fibroblasts to other cell types, such as neurons (Vierbuchen et al., 2010), multilineage blood cells
(Szabo et al., 2010), and cardiomyocytes (leda et al., 2010). Furthermore, somatic cells other than fibroblasts have been directly converted into other cell types, and even direct endoderm-to-ectoderm switching has been achieved (Marro et al., 2011). It has been also demonstrated that fibroblasts can be reprogrammed into neural stem cells (Kim et al., 2011a; Han et al., 2012; Thier et al., 2012). In this review, we will discuss these recent studies in iN cells and iNS cells conversion from terminally differentiated cells by different combinations of transcription factors (Fig. 1), miRNAs and small molecules.

\section{DIRECT REPROGRAMMING OF NON-NEURAL CELLS TO NEURONS}

Many neuronal transcription factors that are involved in cell-fate specification have been identified, and scientists have been able to directly reprogram mouse fibroblasts into neuronal cells by ectopically expressing transcription factors (Fig. 2). The expression of three transcription factors: Ascl1, Brn2, and Myt1I (ABM), which were selected from 19 candidate genes following lentiviral expression, was sufficient to directly convert embryonic and postnatal mouse fibroblasts into functional neurons that express multiple neuron-specific proteins, exhibit action potentials and form functional synapses. The resulting cells were termed induced neuronal (iN) cells (Vierbuchen et al., 2010). Subsequent experiments showed that the majority of iN cells induced by ABM had excitatory neuron phenotypes and exhibited glutamatergic properties.

One possible mechanism underlying the induction from somatic cells to neurons could be that the ectopic expression of neuron-specific transcription factors initiates reprogramming and then further activates neuron-specific downstream target genes. Induced neuronal cells are ultimately produced after neuronal networks are fully activated and stabilized. These ectopic neuron-specific factors might also activate 


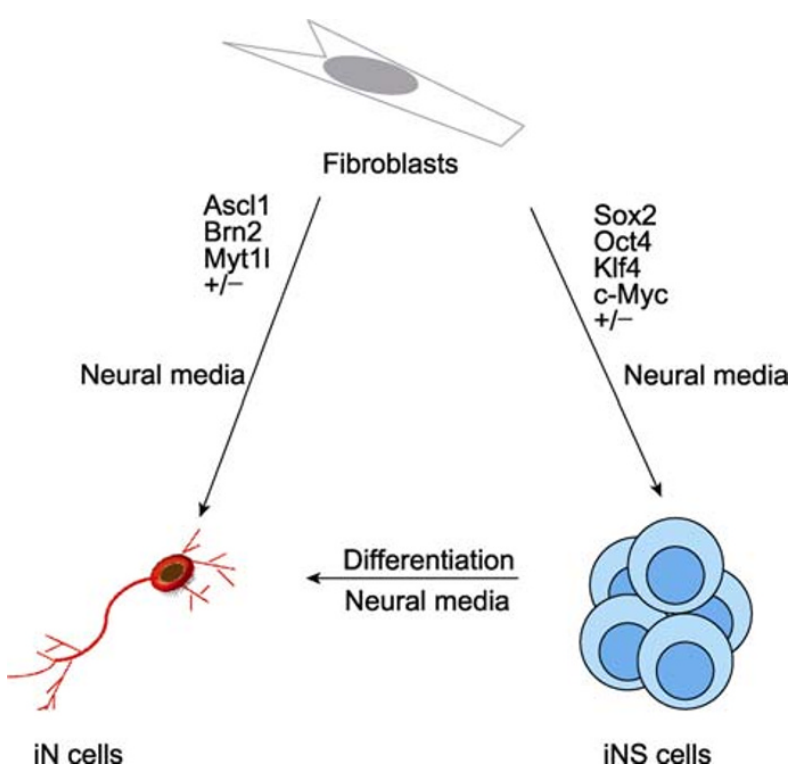

Figure 1. Overview of the direct reprogramming of neural lineage cells. Neural stem cells and subtypes of neurons can be directly induced from fibroblasts and other cells. epigenetic programs, including DNA methylation/demethylation and histone modification, that stabilize the neuronal network (Vierbuchen and Wernig, 2011). It has been speculated that only a small subpopulation of somatic cells can be stimulated by neuronal transcription factors, which might open the transition from one lineage to another (Yamanaka, 2009).

Following the first study of induced neuronal cells from fibroblasts in the mouse, an important open question was whether human induced neuronal cells could be obtained from human fibroblasts. This research issue is crucial; if human iN cells could be produced successfully, they could then be used in cell replacement therapies or other applications. It was thought that human iN cells from human fibroblasts might be generated with the same factors used to convert mouse fibroblasts to iN cells. However, the same combination of factors does not elicit the same outcome in human fibroblasts, generating neurons with limited functional properties (Pang et al., 2011). This result was also confirmed by Qiang et al. (2011) who showed that ABM factors had little effect on reprogramming human cells and instead caused apoptotic cell death. Pang et al. (2011) therefore screened 20 additional factors in combination with $\mathrm{ABM}$ and found that the

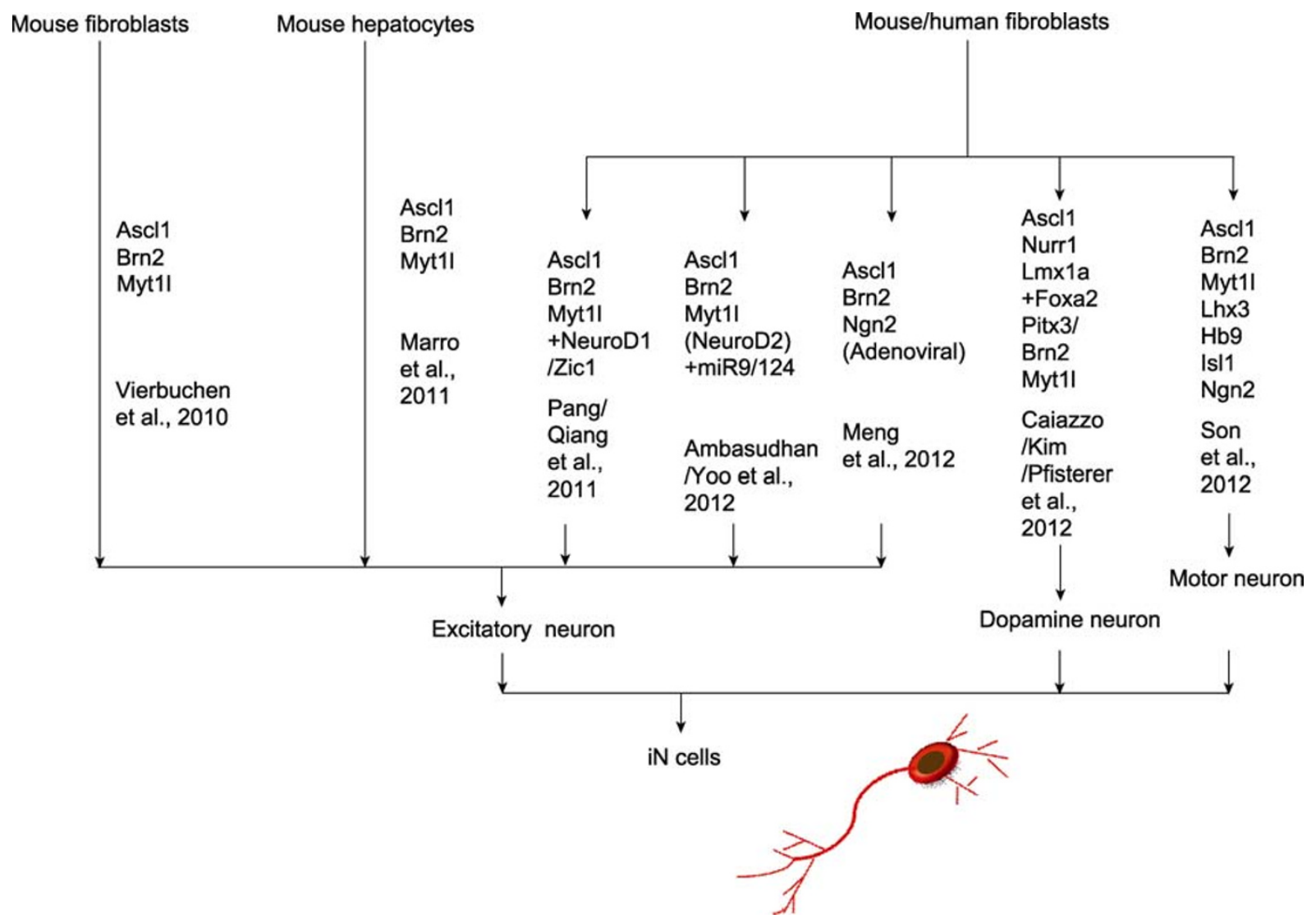

Figure 2. Generation of induced neuronal (iN) cells. Several studies have successfully demonstrated that induced neuronal cells can be obtained from mouse and/or human fibroblasts as well as other types of cells. The diagrams here show examples of induced neuronal cells generated from various types of cells by different combinations of transcription factors or miRNAs. 
co-expression of ABM and the basic helix-loop-helix (bHLH) transcription factor NeuroD1 is sufficient to generate functional neuronal cells from human fibroblasts. The study by Qiang showed the combination of Ascl1, Brn2 and Zic1 is sufficient to induce neuronal cells from AD cells. Myt1l only enhances this conversion. It would be of great interest to investigate whether these transcription factors exhibit cell conversion by interacting with each other, forming multiprotein complexes and binding to common targets.

In addition to transcription factors, microRNAs (miRNAs), which are involved in regulating target gene activity, seem to play important roles in cell reprogramming and cell fate determination. Now that certain microRNAs are known to contribute to neuronal fates, the next question is whether their activity could complement the activities of transcription factors in the conversion process. In most cases, reprogramming factors are known as transcriptional activators, while miRNAs typically act through the direct repression of multiple genes. Thus, it is conceivable that miRNAs provide a direct and effective repressive action targeting a specific set of genes that are not directly bound by the transcription factors and must be inhibited for improved and accelerated cell reprogramming. Further evidence demonstrates that miR-9/9* and miR-124 regulate gene expression and promote a pervasive chromatin remodeling process (Yoo et al., 2009). Therefore, brain-specific miRNAs might promote the conversion process from fibroblasts to iN cells. Through a hypothesis-driven approach, two research groups (Ambasudhan et al., 2011; Yoo et al., 2011) revealed that miRNAs act with neuron-specific transcription factors to drive the conversion of human fibroblasts into neurons. Ambasudhan et al. discovered that miR-124 could induce iN cells conversion by combining with Brn2 and Myt1l. Surprisingly, this group also showed that Ascl1 was dispensable for direct neuronal reprogramming (Ambasudhan et al., 2011). Yoo et al. (2011) further found that an miRNA cocktail combining miR-124 and miR-9/9* had a strong cooperative effect in neuronal conversion. Indeed, both miR-9/ $9^{*}$ and miR-124 are co-expressed in post-mitotic neurons. These factors were previously found to promote neuronal differentiation and were reported to downregulate Baf53a expression (Shibata et al., 2011).

For the clinical application of induced neuronal cells, it would be very useful to generate specific subtypes of induced neuronal cells from fibroblasts. Since the initial findings in iN cells, new research has focused on whether region-specific and neurotransmitter-specific neurons could be obtained. In addition to Vierbuchen's ABM-driven excitatory neurons, Caiazzo et al. (2011) found another set of three transcription factors (Ascl1, Nurr1 and Lmx1a) that can, in combination, directly convert mouse and human fibroblasts to dopaminergic neurons. In addition, Pfisterer et al. (2011) discovered that ABM combined with two dopaminergic-specific neuron transcription factors, Lmx1a and FoxA2, could induce human fibroblasts to become dopamine neurons. Kim et al. (2011b) directed the phenotypes of mouse and human fibroblasts cells toward Pitx3+ neurons that closely resemble midbrain dopaminergic neurons. Subtype-specific iN cells derived from human fibroblasts could be used for brain disease therapy and drug screening. Son et al. reported that the ectopic expression of seven transcription factors (Ascl1, Brn2, Myt1 I, Lhx3, Hb9, Isl1 and Ngn2) could induce mouse and human fibroblasts to become motor neurons (Son et al., 2011). Marro et al. (2011) reported the direct reprogramming of definitive endodermal cells (mouse hepatocytes) to induced neuronal cells with the same ABM factors, indicating that lineage conversion through forced expression of transcription factors is possible in any cell type.

Furthermore, it is noteworthy that the transcription profile of the initial cell type was strongly inhibited in both fibroblastand hepatocyte-derived iN cells. This finding demonstrates that the same combination of neuronal transcription factors could activate the neuronal program and simultaneously silence the initial non-neuronal cell transcriptional program (Vierbuchen et al., 2010; Marro et al., 2011; Yang et al., 2011) and that the same transcription factors could produce similar induced neuronal cells from different cell types. However, although the initial transcriptional profile was silenced and neuronal profile acquired, these iN cells do possess some epigenetic memories of their cells of origin (Marro et al., 2011). It will be interesting to further investigate when and whether these original cells memories are ultimately turned off and whether these memories affect the comprehensive functions of converted neurons.

Although these conversions are efficient and functional, all of the studies in this field have employed retroviral or lentiviral vector delivery systems. These viruses may integrate into the genome and cause insertional mutations or the activation of tumor-related genes, thus limiting their application. Therefore, it is desirable to generate neurons with a non-integrating system. Our group used non-integrating adenoviruses carrying with a different combination of transcription factors, Ascl1, Brn2 and Ngn2 (ABN), to directly convert mouse embryonic and adult fibroblasts to functional neurons (Meng et al., 2012). Ngn2 alone had previously been reported to direct the development of astrocytes into neurons (Berninger et al., 2007; Heinrich et al., 2010). This non-integrating delivery system could provide an ideal prospect for iN cells application in regenerative medicine.

\section{ENHANCING IN CELLS REPROGRAMMING EFFICIENCY}

Currently, it is possible to generate iN cells using transcription factors, but the first ABM-derived mature iN cells are converted with efficiencies of only $20 \%$. Therefore, the development of methods to increase the efficiency of reprogramming seems to be the next urgently needed step. Because neurons are non-dividing cells, reprogramming efficiency is 
an especially important issue in this conversion system.

In the reprogramming of induced pluripotent stem cells (iPSCs), researchers determined that several genes could enhance the efficiency of the reprogramming process. The transient inhibition of the p53 pathway renders iPS cell generation more efficient in terms of the number of cells reprogrammed (Kawamura et al., 2009; Neveu et al., 2010; Okita et al., 2011), while the coexpression of Rarg (retinoid acid receptor- $\gamma$ ) and Lrh-1 (liver receptor homologue 1, also known as orphan nuclear receptor $\mathrm{Nr5a2}$ ) with the four iPS cell factors greatly promoted the reprogramming efficiency (Wang et al., 2011). Furthermore, Nr5a2 can replace Oct4 in the derivation of iPS cells from mouse fibroblasts and also enhances reprogramming efficiency (Heng et al., 2010). These findings demonstrated that signaling through RARs plays critical roles in cellular reprogramming and that the synergistic interaction between Rarg and Lrh1 accelerates the reprogramming process. Based on these data, we presume that these genes may have some role in inducing the neural lineage. Surprisingly, we found that these two factors (Rarg and Lrh1) were significantly effective in induced neuron cells in a recent study (unpublished data).

In addition to those factors, small molecules that target-specific signaling pathways and genes have been reported to be particularly involved in manipulating cell differentiation, cell state and function (Zhu et al., 2011). Several research groups have investigated whether conversion efficiency can be increased by small chemical compounds together with a minimum number of transcription factors. Ladewig et al. (2012) recently reported that two transcription factors (Ascl1 and Ngn2) in combination with three small compounds (SM cocktail-CHIR, SB and noggin) could convert human fibroblasts to iN cells with high yields and neuronal purities up to $>80 \%$, compared to a conversion ratio below $10 \%$ without the small compound cocktail of Ladewig. The SM cocktail inhibits glycogen synthase kinase-3b and the SMAD pathway, both of those inhibition have been shown to be involved in the highly efficient neural differentiation induction from human embryonic stem cells and iPS cells (Chambers et al., 2009; Li et al., 2010). In this study, approximately $35 \%$ of the iN cells were excitatory glutamatergic neurons expressing vesicular glutamate transporter 1 (vGLUT1), 20\% were inhibitory neurons expressing $\mathrm{Y}$-aminobutyric acid (GABA) and $5 \%$ were dopaminergic neurons that were positive for serotonin and tyrosine hydroxylase. However, there is no expression of choline acetyltransferase (cholinergic neuron marker) or HB9 (motor neuron marker) (Ladewig et al., 2012), indicating that those iN cells retain the capacity for probabilistic differentiation. Small molecules are not only useful for enhancing the generation of the desired cell types in vitro but also beneficial for the clinical application and regeneration of endogenous cells in vivo. Many studies have verified that specific small molecules can substitute for a corresponding transcription factor (Shi et al.,
2008; Ichida et al., 2009; Zhu et al., 2011). The next step in this line of research will be to screen libraries of small molecules for compounds that can mimic the effects of transcription factors and investigate their mechanisms of action.

\section{DIRECT REPROGRAMMING OF NON-NEURAL CELLS TO NEURAL STEM CELLS}

Although iN cells could be beneficial in neural development and cell replacement studies, their postmitotic properties confer several disadvantages, namely the inability to proliferate and differentiate into needed cell types. Thus, it is necessary to develop techniques to obtain induced neural stem/progenitor (iNS) cells for cell differentiation, cell therapy, and drug discovery (Liu et al., 2012) (Fig. 3).

Kim et al. (2011a) investigated whether iNS cells could be generated from somatic cells by reprogramming cells with the same four reprogramming factors used to generate iPS cells followed by culture in neural stem (NS) cells medium. They successfully reprogrammed fibroblasts to iNS cells over an abbreviated induction period, and these iNS cells expressed several NS cell-specific markers, including promyelocytic leukemia zinc finger (Plzf), a rosette NSC marker (Elkabetz et al., 2008), and Pax6, an early neural transcription factor (Walther and Gruss, 1991). Various mature neuronal and glial markers were also detected in those induced NS cells after the spontaneous differentiation of cells from isolated colonies. Compared with the circuitous two-step strategy from somatic cells to iPS cells and subsequent development of iPS cells to NS cells, this straightforward iNS cell reprogramming method is highly efficient, direct, and rapid; it requires only one step that is completed within 2 weeks and yields almost $100 \%$ colonies of NS cells. Compared with iN cells, reprogrammed iNS cells also have distinct advantages in that can could be expanded in vitro and retain the ability to differentiate into multiple subtypes of neurons and glial cells. The success of this direct reprogramming of iNS cells provides a unique paradigm for the generation of iNS cells using iPS factors in a modified period and culture medium, and a similar strategy could be used for reprogramming other cell lineages.

More recently, several independent groups have shown that a combination of transcription factors could directly reprogram mouse fibroblasts toward stably expandable induced neural stem cells (Han et al., 2012; Thier et al., 2012). These studies present alternative strategies for reprogramming somatic cells to neural stem cell identity. Thier et al. used the four 'classical' iPS reprogramming factors (Sox2, Klf4, c-Myc and Oct4) but limited the expression of Oct4 during the initial period of reprogramming. The generated iNS cells form neurosphere-like colonies and can be expanded for more than 50 passages without the sustained expression of iPS cell factors (Thier et al., 2012). These iNS cells exhibit morphological and molecular characters of NS cells, express multiple NS cells markers (include Nestin, Pax6, and Olig2) 


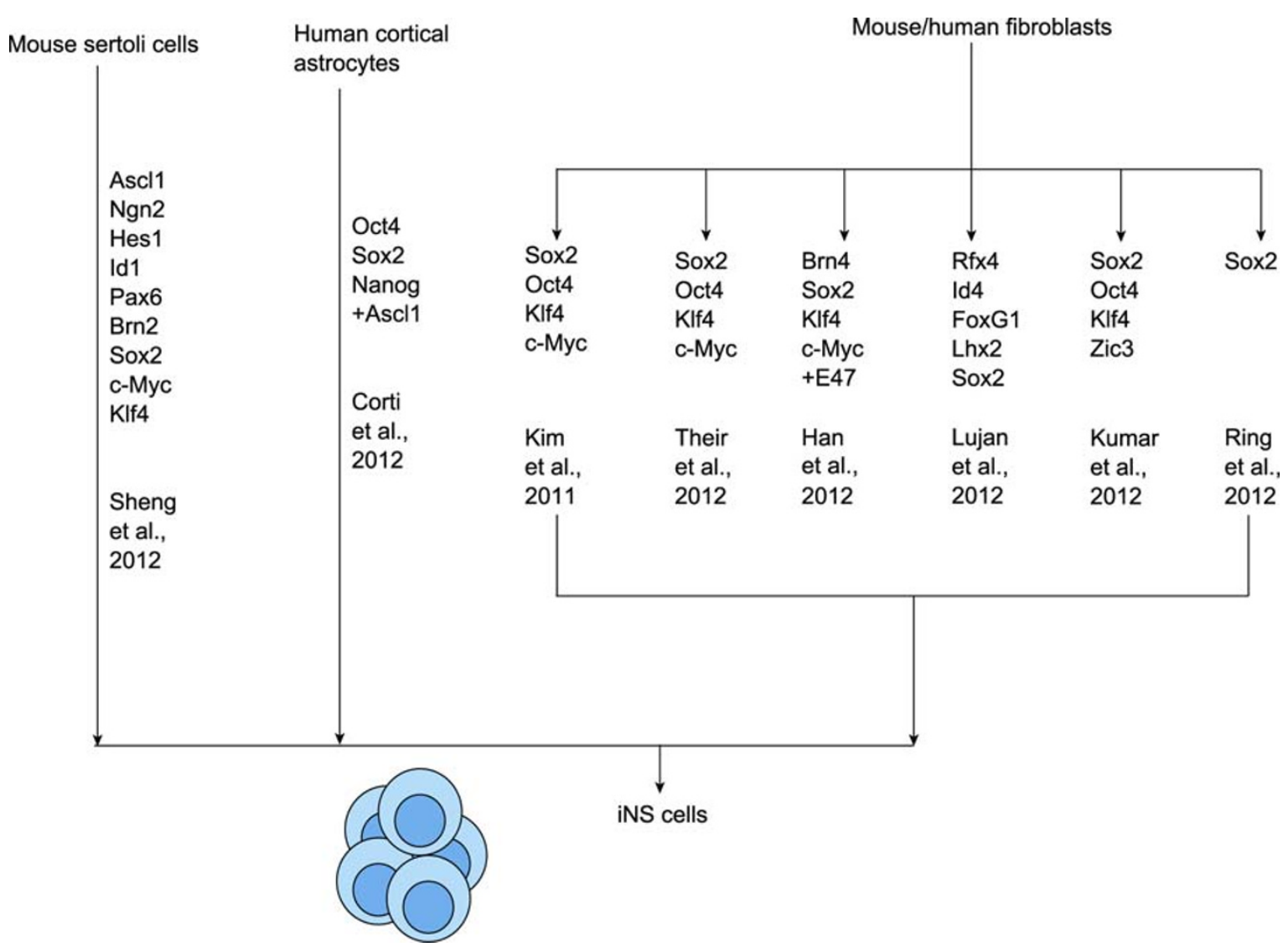

Figure 3. Generation of induced neural stem (iNS) cells. Several studies have demonstrated that induced neural stem/precursor cells can be obtained from mouse and/or human fibroblasts as well as other types of cells. These diagrams show examples of induced self-renewing and multipotent neural stem cells generated from various cell types by different combinations of transcription factors.

and have an identity similar to that of endogenous brainderived NS cells. Moreover, iNS cells can be differentiated into neurons, astrocytes, and oligodendrocytes in vitro. These results demonstrate that functional iNS cells can be generated from somatic cells with stably expandable features by transcription factors that drive reprogramming.

Han et al. showed that reprogramming with a combination of transcription factors (Brn4/Pou3f4, Sox2, KIf4, c-Myc, plus E47/Tcf3) directly induced neural stem cell identity in mouse fibroblasts and that iNS cells did not generate teratomas after injection into immunosuppressed mice (Han et al., 2012). The multipotent iNS cells display NS cell morphology, differentiation capacity, and self-renewing potential with gene expression features and epigenetic profiles similar to those of brain-derived NS cells. Moreover, the cells grafted after in vivo differentiation had committed to the neuronal lineage, and only a small percentage of the transplanted cells maintained a neural progenitor identity for an extended period. All of these results indicate that somatic cells can be reprogrammed into stably expandable iNS cells with defined transcription factors.
Lujan et al. recently reported the generation of self-renewing induced neural precursor (iNP) cells from mouse embryonic fibroblasts using five factors (Rfx4, ID4, FoxG1, Lhx2, and Sox2) (Lujan et al., 2012). The transfection of these factors into mouse embryonic fibroblasts (MEFs) was sufficient to induce Sox2-EGFP+ colonies, as assessed $24 \mathrm{~d}$ after infection. With stepwise elimination experiments, these authors found that the combination of Sox2 and FoxG1 can generate self-renewed iNP cells that have the capacity to differentiate to functional neurons and astroglial cells. The addition of Brn2 to Sox2 and FoxG1 helped to generate multipotent NP cells that can be differentiated into all cell types in neural systems.

Because the oncogene cMyc may cause cell excess proliferation or carcinogenesis, the use of cMyc limits the application of artificially induced cells. Kumar et al. demonstrated that the co-transduction of Zic3 with Oct4, Sox2, and KIf4 could convert human fibroblasts to iNS cells. Zic3, a member of the Gli superfamily, has been implicated in the maintenance of pluripotency in mouse and human embryonic stem cells (ESCs), where it directly controls the ex- 
pression of Nanog (Lim et al., 2007, 2010). Zic3 is also an immediate early gene induced by fibroblast growth factor (FGF) signaling during neural specification (Marchal et al., 2009). By preventing neuronal differentiation, Zic3 plays a role in the maintenance of the neural progenitor cell fate (Inoue et al., 2007). These studies demonstrate that Zic3 plays important roles in the commitment to and maintenance of iNP cell identity. One potential outcome of these experiments is the creation of stable iNP cells lines (Kumar et al., 2012).

Like iN cells, iNS cells may also be generated from various origin cell types. In addition to fibroblasts, human cortical astrocytes could be induced into the neural stem/progenitor phenotype to obtain progenitor and mature cells with a neural fate through the ectopic expression of the reprogramming factors Oct4, Sox2, and Nanog, and the additional expression of Ascl1 further promoted neuronal phenotype acquisition in vitro and in vivo (Corti et al., 2012). Interestingly, pup Sertoli cells were also transformed into multipotent neural stem cells by the expression of nine transcription factors (Ascl1, Ngn2, Hes1, Id1, Pax6, Brn2, Sox2, c-Myc, and Klf4) that are expressed in brain neural stem/progenitor cells (Sheng et al., 2012). Surprisingly, the only transcription factor that was dispensable for this reprogramming was Sox2; the eight remaining factors (Ascl1, Ngn2, Hes1, Id1, Pax6, Brn2, c-Myc, and Klf4) were required to generate iNS cells colonies, implying a complex reprogramming process between these two lineages.

Because clinical applications must consider the risk of tumor formation and the self-renewability in culture, iNS cells would be preferred due to their simplicity. A recent report demonstrated the generation of iNS cells from mouse and human fibroblasts by direct reprogramming with a single factor, Sox2 (Ring et al., 2012). The derived NSC-like cells expressed Sox2, Nestin, Sox1, and Zbtb16 but did not express pluripotency-related genes such as Oct4, Nanog, and Zfp42; further, these cells can survive, integrate, and differentiate in vivo and do not generate tumors. Thus, Sox2 might be used in combination with other factors to create neural progenitors that can develop into subtype-specific neurons, which would be invaluable for mechanistic studies, drug screening, and potential cell therapies for different neurodegenerative diseases.

More about the potential functional capacity of microRNAs in iNSC reprogramming could be learned from research in iPS cells. It was reported that mouse and human iPS cells can be generated by a combination of miRNAs and transcription factors (Miyoshi et al., 2011). The mouse embryonic stem cell-specific microRNAs (miRNA) miR-291-3p, miR-294, and miR-295 have been shown to enhance the efficiency of Klf4, Oct4 and Sox2-induced pluripotency (Judson et al., 2009). Therefore, NS cell-specific miRNAs may be screened and investigated to drive induced neural stem cell generation.

\section{PERSPECTIVE}

The first iN cell study open another line of reprogramming research in a new era following iPS cell discovery. In contrast to iPS cell induction, iN cell reprogramming does not require a neural progenitor state; instead, it involves the direct conversion from one lineage to another different lineage, bypassing the proliferation state (Hong et al., 2009; Kawamura et al., 2009; Li et al., 2009; Marion et al., 2009; Utikal et al., 2009). Moreover, the reprogramming of iN cells provides a new approach to the study of cell fate determination, drug discovery, and cell replacement. Following iN cell studies, iNS cell reprogramming further pushes reprogramming research to a higher level for central nervous system research. iNS cells have several advantages for cell therapies and cell replacement, namely that they can be expanded in vitro and further be differentiated into specific types of neurons. It will be interesting to study which signaling pathways are activated during the reprogramming process and which endogenous transcription factors and epigenetic factors are involved in the switch from one cell type to another type or lineage.

The studies of iN and iNS cells could advance human brain research because it is not applicable to obtain endogenous neural stem cells from human brains. Additionally, iN cells generated from brain disease patients could be used for neural modeling as well as to simulate the stage at which these neurons become disordered in vivo and to determine which mutant genes cause the disease phenotype (i.e., functional loss).

Although both iN and iNS cells approaches must be optimized to obtain high reprogramming efficiency and subtype cell specification, direct conversion has challenged the traditional concepts and provided another approach to the study of neural development. With respect to potential translational research, iN or iNS cells might be used for clinical applications in the treatment of neurological disease. Future studies will seek to increase the efficacy of iN and iNS cell generation and to generate stable and safe cells without virus integration from adult human fibroblasts before translational applications can become available.

\section{ACKNOWLEDGEMENTS}

This work was supported by the National Basic Research Program (973 Program) (No. 2011CBA00402, J.J.), the "Strategic Priority Research Program" of the Chinese Academy of Sciences (No. XDA01020301, J.J.), and the Hundred Talent Program (J.J.).

\section{ABBREVIATIONS}

ESCs, embryonic stem cells; GABA, Y-aminobutyric acid; iN cells, induced neuronal cells; iNP cells, induced neural progenitor cells; iNS cells, induced neural stem cells; iPS cells, induced pluripotent stem 
cells; MEFs, mouse embryonic fibroblasts; miR, microRNAs; NSCs, neural stem cells; vGLUT1, vesicular glutamate transporter 1

\section{REFERENCES}

Ambasudhan, R., Talantova, M., Coleman, R., Yuan, X., Zhu, S., Lipton, S.A., and Ding, S. (2011). Direct reprogramming of adult human fibroblasts to functional neurons under defined conditions. Cell Stem Cell 9, 113-118.

Berninger, B., Costa, M.R., Koch, U., Schroeder, T., Sutor, B., Grothe, B., and Gotz, M. (2007). Functional properties of neurons derived from in vitro reprogrammed postnatal astroglia. J Neurosci 27, 8654-8664.

Caiazzo, M., Dell'Anno, M.T., Dvoretskova, E., Lazarevic, D., Taverna, S., Leo, D., Sotnikova, T.D., Menegon, A., Roncaglia, P., Colciago, G., et al. (2011). Direct generation of functional dopaminergic neurons from mouse and human fibroblasts. Nature 476, 224-227.

Chambers, S.M., Fasano, C.A., Papapetrou, E.P., Tomishima, M., Sadelain, M., and Studer, L. (2009). Highly efficient neural conversion of human ES and iPS cells by dual inhibition of SMAD signaling. Nat Biotechnol 27, 275-280.

Corti, S., Nizzardo, M., Simone, C., Falcone, M., Donadoni, C., Salani, S., Rizzo, F., Nardini, M., Riboldi, G., Magri, F., et al. (2012). Direct reprogramming of human astrocytes into neural stem cells and neurons. Exp Cell Res 318, 1528-1541.

Davis, R.L., Weintraub, H., and Lassar, A.B. (1987). Expression of a single transfected cDNA converts fibroblasts to myoblasts. Cell 51, 987-1000.

Elkabetz, Y., Panagiotakos, G., Al Shamy, G., Socci, N.D., Tabar, V., and Studer, L. (2008). Human ES cell-derived neural rosettes reveal a functionally distinct early neural stem cell stage. Genes Dev 22, 152-165.

Han, D.W., Tapia, N., Hermann, A., Hemmer, K., Hoing, S., Arauzo-Bravo, M.J., Zaehres, H., Wu, G., Frank, S., Moritz, S., et al. (2012). Direct reprogramming of fibroblasts into neural stem cells by defined factors. Cell Stem Cell 10, 465-472.

Heinrich, C., Blum, R., Gascon, S., Masserdotti, G., Tripathi, P., Sanchez, R., Tiedt, S., Schroeder, T., Gotz, M., and Berninger, B. (2010). Directing astroglia from the cerebral cortex into subtype specific functional neurons. PLoS Biol 8, e1000373.

Heng, J.C., Feng, B., Han, J., Jiang, J., Kraus, P., Ng, J.H., Orlov, Y.L., Huss, M., Yang, L., Lufkin, T., et al. (2010). The nuclear receptor Nr5a2 can replace Oct4 in the reprogramming of murine somatic cells to pluripotent cells. Cell Stem Cell 6, 167-174.

Hong, H., Takahashi, K., Ichisaka, T., Aoi, T., Kanagawa, O., Nakagawa, M., Okita, K., and Yamanaka, S. (2009). Suppression of induced pluripotent stem cell generation by the p53-p21 pathway. Nature 460, 1132-1135.

Ichida, J.K., Blanchard, J., Lam, K., Son, E.Y., Chung, J.E., Egli, D., Loh, K.M., Carter, A.C., Di Giorgio, F.P., Koszka, K., et al. (2009). A small-molecule inhibitor of tgf-Beta signaling replaces sox2 in reprogramming by inducing nanog. Cell Stem Cell 5, 491-503.

leda, M., Fu, J.D., Delgado-Olguin, P., Vedantham, V., Hayashi, Y., Bruneau, B.G., and Srivastava, D. (2010). Direct reprogramming of fibroblasts into functional cardiomyocytes by defined factors. Cell 142, 375-386.
Inoue, T., Ota, M., Ogawa, M., Mikoshiba, K., and Aruga, J. (2007). Zic1 and Zic3 regulate medial forebrain development through expansion of neuronal progenitors. J Neurosci 27, 5461-5473.

Judson, R.L., Babiarz, J.E., Venere, M., and Blelloch, R. (2009). Embryonic stem cell-specific microRNAs promote induced pluripotency. Nat Biotech 27, 459-461.

Kawamura, T., Suzuki, J., Wang, Y.V., Menendez, S., Morera, L.B., Raya, A., Wahl, G.M., and Izpisua Belmonte, J.C. (2009). Linking the p53 tumour suppressor pathway to somatic cell reprogramming. Nature 460, 1140-1144.

Kim, J., Efe, J.A., Zhu, S., Talantova, M., Yuan, X., Wang, S., Lipton, S.A., Zhang, K., and Ding, S. (2011a). Direct reprogramming of mouse fibroblasts to neural progenitors. Proc Natl Acad Sci U S A 108, 7838-7843.

Kim, J., Su, S.C., Wang, H., Cheng, A.W., Cassady, J.P., Lodato, M.A., Lengner, C.J., Chung, C.Y., Dawlaty, M.M., Tsai, L.H., et al. (2011b). Functional integration of dopaminergic neurons directly converted from mouse fibroblasts. Cell Stem Cell 9, 413-419.

Kumar, A., Declercq, J., Eggermont, K., Agirre, X., Prosper, F., and Verfaillie, C.M. (2012). Zic3 induces conversion of human fibroblasts to stable neural progenitor-like cells. J Mol Cell Biol 4, 252-255.

Ladewig, J., Mertens, J., Kesavan, J., Doerr, J., Poppe, D., Glaue, F., Herms, S., Wernet, P., Kogler, G., Muller, F.J., et al. (2012). Small molecules enable highly efficient neuronal conversion of human fibroblasts. Nat Methods 9, 575-578.

Li, H., Collado, M., Villasante, A., Strati, K., Ortega, S., Canamero, M., Blasco, M.A., and Serrano, M. (2009). The Ink4/Arf locus is a barrier for iPS cell reprogramming. Nature 460, 1136-1139.

Li, R., Liang, J., Ni, S., Zhou, T., Qing, X., Li, H., He, W., Chen, J., Li, F., Zhuang, Q., et al. (2010). A mesenchymal-to-epithelial transition initiates and is required for the nuclear reprogramming of mouse fibroblasts. Cell Stem Cell 7, 51-63.

Lim, L.S., Hong, F.H., Kunarso, G., and Stanton, L.W. (2010). The pluripotency regulator Zic3 is a direct activator of the Nanog promoter in ESCs. Stem Cells 28, 1961-1969.

Lim, L.S., Loh, Y.H., Zhang, W., Li, Y., Chen, X., Wang, Y., Bakre, M., Ng, H.H., and Stanton, L.W. (2007). Zic3 is required for maintenance of pluripotency in embryonic stem cells. Mol Biol Cell 18, 1348-1358.

Liu, G.H., Yi, F., Suzuki, K., Qu, J., and Izpisua Belmonte, J.C. (2012). Induced neural stem cells: a new tool for studying neural development and neurological disorders. Cell Res 22, 1087-1091.

Lujan, E., Chanda, S., Ahlenius, H., Sudhof, T.C., and Wernig, M. (2012). Direct conversion of mouse fibroblasts to self-renewing, tripotent neural precursor cells. Proc Natl Acad Sci U S A 109, 2527-2532.

Marchal, L., Luxardi, G., Thome, V., and Kodjabachian, L. (2009). BMP inhibition initiates neural induction via FGF signaling and Zic genes. Proc Natl Acad Sci U S A 106, 17437-17442.

Marion, R.M., Strati, K., Li, H., Murga, M., Blanco, R., Ortega, S., Fernandez-Capetillo, O., Serrano, M., and Blasco, M.A. (2009). A p53-mediated DNA damage response limits reprogramming to ensure iPS cell genomic integrity. Nature 460, 1149-1153.

Marro, S., Pang, Z.P., Yang, N., Tsai, M.C., Qu, K., Chang, H.Y., Sudhof, T.C., and Wernig, M. (2011). Direct lineage conversion of 
terminally differentiated hepatocytes to functional neurons. Cell Stem Cell 9, 374-382.

Meng, F., Chen, S., Miao, Q., Zhou, K., Lao, Q., Zhang, X., Guo, W., and Jiao, J. (2012). Induction of fibroblasts to neurons through adenoviral gene delivery. Cell Res 22, 436-440.

Miyoshi, N., Ishii, H., Nagano, H., Haraguchi, N., Dewi, D.L., Kano, Y., Nishikawa, S., Tanemura, M., Mimori, K., Tanaka, F., et al. (2011). Reprogramming of mouse and human cells to pluripotency using mature microRNAs. Cell Stem Cell 8, 633-638.

Neveu, P., Kye, M.J., Qi, S., Buchholz, D.E., Clegg, D.O., Sahin, M., Park, I.H., Kim, K.S., Daley, G.Q., Kornblum, H.I., et al. (2010). MicroRNA profiling reveals two distinct p53-related human pluripotent stem cell states. Cell Stem Cell 7, 671-681.

Okita, K., Matsumura, Y., Sato, Y., Okada, A., Morizane, A., Okamoto, S., Hong, H., Nakagawa, M., Tanabe, K., Tezuka, K., et al. (2011). A more efficient method to generate integration-free human iPS cells. Nat Methods 8, 409-412.

Pang, Z.P., Yang, N., Vierbuchen, T., Ostermeier, A., Fuentes, D.R., Yang, T.Q., Citri, A., Sebastiano, V., Marro, S., Sudhof, T.C., et al. (2011). Induction of human neuronal cells by defined transcription factors. Nature 476, 220-223.

Pfisterer, U., Kirkeby, A., Torper, O., Wood, J., Nelander, J., Dufour, A., Bjorklund, A., Lindvall, O., Jakobsson, J., and Parmar, M. (2011). Direct conversion of human fibroblasts to dopaminergic neurons. Proc Natl Acad Sci U S A 108, 10343-10348.

Qiang, L., Fujita, R., Yamashita, T., Angulo, S., Rhinn, H., Rhee, D., Doege, C., Chau, L., Aubry, L., Vanti, W.B., et al. (2011). Directed conversion of Alzheimer's disease patient skin fibroblasts into functional neurons. Cell 146, 359-371.

Ring, K.L., Tong, L.M., Balestra, M.E., Javier, R., Andrews-Zwilling, Y., Li, G., Walker, D., Zhang, W.R., Kreitzer, A.C., and Huang, Y. (2012). Direct reprogramming of mouse and human fibroblasts into multipotent neural stem cells with a single factor. Cell Stem Cell 11, 100-109.

Sheng, C., Zheng, Q., Wu, J., Xu, Z., Wang, L., Li, W., Zhang, H., Zhao, X.Y., Liu, L., Wang, Z., et al. (2012). Direct reprogramming of Sertoli cells into multipotent neural stem cells by defined factors. Cell Res 22, 208-218.

Shi, Y., Desponts, C., Do, J.T., Hahm, H.S., Scholer, H.R., and Ding, S. (2008). Induction of pluripotent stem cells from mouse embryonic fibroblasts by Oct4 and Klf4 with small-molecule compounds. Cell Stem Cell 3, 568-574.

Shibata, M., Nakao, H., Kiyonari, H., Abe, T., and Aizawa, S. (2011). MicroRNA-9 regulates neurogenesis in mouse telencephalon by targeting multiple transcription factors. J Neurosci 31, 3407-3422.

Son, E.Y., Ichida, J.K., Wainger, B.J., Toma, J.S., Rafuse, V.F.,
Woolf, C.J., and Eggan, K. (2011). Conversion of mouse and human fibroblasts into functional spinal motor neurons. Cell Stem Cell 9, 205-218.

Szabo, E., Rampalli, S., Risueno, R.M., Schnerch, A., Mitchell, R., Fiebig-Comyn, A., Levadoux-Martin, M., and Bhatia, M. (2010). Direct conversion of human fibroblasts to multilineage blood progenitors. Nature 468, 521-526.

Thier, M., Worsdorfer, P., Lakes, Y.B., Gorris, R., Herms, S., Opitz, T., Seiferling, D., Quandel, T., Hoffmann, P., Nothen, M.M., et al. (2012). Direct conversion of fibroblasts into stably expandable neural stem cells. Cell Stem Cell 10, 473-479.

Utikal, J., Polo, J.M., Stadtfeld, M., Maherali, N., Kulalert, W., Walsh, R.M., Khalil, A., Rheinwald, J.G., and Hochedlinger, K. (2009). Immortalization eliminates a roadblock during cellular reprogramming into iPS cells. Nature 460, 1145-1148.

Vierbuchen, T., Ostermeier, A., Pang, Z.P., Kokubu, Y., Sudhof, T.C., and Wernig, M. (2010). Direct conversion of fibroblasts to functional neurons by defined factors. Nature 463, 1035-1041.

Vierbuchen, T., and Wernig, M. (2011). Direct lineage conversions: unnatural but useful? Nat Biotech 29, 892-907.

Walther, C., and Gruss, P. (1991). Pax-6, a murine paired box gene, is expressed in the developing CNS. Development 113, 1435-1449.

Wang, W., Yang, J., Liu, H., Lu, D., Chen, X., Zenonos, Z., Campos, L.S., Rad, R., Guo, G., Zhang, S., et al. (2011). Rapid and efficient reprogramming of somatic cells to induced pluripotent stem cells by retinoic acid receptor gamma and liver receptor homolog 1 . Proc Natl Acad Sci U S A 108, 18283-18288.

Yamanaka, S. (2009). Elite and stochastic models for induced pluripotent stem cell generation. Nature 460, 49-52.

Yamanaka, S., and Takahashi, K. (2006). [Induction of pluripotent stem cells from mouse fibroblast cultures]. Tanpakushitsu kakusan koso. Protein, nucleic acid, enzyme 51, 2346-2351.

Yang, N., Ng, Y.H., Pang, Z.P., Sudhof, T.C., and Wernig, M. (2011). Induced neuronal cells: how to make and define a neuron. Cell Stem Cell 9, 517-525.

Yoo, A.S., Staahl, B.T., Chen, L., and Crabtree, G.R. (2009). MicroRNA-mediated switching of chromatin-remodelling complexes in neural development. Nature 460, 642-646.

Yoo, A.S., Sun, A.X., Li, L., Shcheglovitov, A., Portmann, T., Li, Y., Lee-Messer, C., Dolmetsch, R.E., Tsien, R.W., and Crabtree, G.R. (2011). MicroRNA-mediated conversion of human fibroblasts to neurons. Nature 476, 228-231.

Zhu, S., Wei, W., and Ding, S. (2011). Chemical strategies for stem cell biology and regenerative medicine. Annu Rev Biomed Eng 13, 73-90. 